\title{
Modified Local and Global Contrast Enhancement Algorithm for Color Satellite Image
}

\author{
Viacheslav Voronin ${ }^{1,2}$ \\ ${ }^{1}$ Moscow State Technological University "STANKIN", RU-127055, Moscow, Russia \\ ${ }^{2}$ Don Sate Technical University, RU-344000, Rostov-on-Don, Russia
}

\begin{abstract}
The quality of remotely sensed satellite images depends on the reflected electromagnetic radiation from the earth's surface features. Lack of consistent and similar amounts of energy reflected by different features from the earth's surface results in a poor contrast satellite image. Image enhancement is the image processing of improving the quality that the results are more suitable for display or further image analysis. In this paper, we present a detailed model for color image enhancement using the quaternion framework. We introduce a novel quaternionic frequency enhancement algorithm that can combine the color channels and the local and global image processing. The basic idea is to apply the $\alpha$-rooting image enhancement approach for different image blocks. For this purpose, we split image in moving windows on disjoint blocks. The parameter alfa for every block and the weights for every local and global enhanced image driven through optimization of measure of enhancement (EMEC). Some presented experimental results illustrate the performance of the proposed approach on color satellite images in comparison with the state-of-the-art methods.
\end{abstract}

\section{Introduction}

The atmospheric influences and sensor characteristics result in a poor contrast on satellite images. So, these images may not be optimized for visual interpretation. Contrast enhancement is an important low-level computer vision technique, which can improve the visibility of the considered images. This kind of algorithm has been widely applied in-camera photographing, video quality improvement, medical imaging, and remote sensing imaging. The principal objective of image enhancement is to modify attributes of an image to make it more suitable for a given task and a specific observer.

All information in the image can only be stored in a small region of colors, which makes it difficult to extract some useful information [1]. The contrast enhancement methods can be divided to follow groups [2-6]:

1) Local enhancement.

In general, for these methods used local window which slides through every pixel for modified contrast in these regions. So, the local contrast can be improved in every region of the image.

2) Global enhancement.

In this type of contrast enhancement techniques, the global histogram information is considered for enhancement.

The histogram equalization (HE) is a widely used method of image enhancement, which transform the spatial histogram. The $\mathrm{HE}$ leads to following disadvantages: the removal of fine details, over- enhancement of the image contrast when high peaks exist on the histogram, an undesired loss of visual data and quality [7].

Adaptive histogram equalization (AHE) is an image processing technique related to a non-uniform spatial improvement technique. This method does not work well with smooth areas and does not eliminate the noise gain in these areas [8]. One of the most popular modifications of AHE is the contrast limited adaptive histogram equalization (CLAHE) [9]. This method has overenhancement resulting in the loss of some local information [10].

The Retinex is effective enhancement method has been proposed in [11]. The enhancement result in this method is not natural for many real type scenes in case of irregular lighting and brightness gradient.

The alpha-rooting approach based on the manipulation of spectral coefficients $[12,13]$. The main disadvantage of this algorithm is the difficulty in choosing the value of the alpha parameter.

The color image enhancement approaches are based on the processing of each color channel (red, green, and blue) separately [14]. However, this methodology fails to capture the inherent correlation between the components and results in color artefacts [14]. The color image enhancement is sometimes leads to undesirable color shifts.

The common problem of all existing image enhancement approaches is to focus on the processing of a specific image property; therefore, it is necessary to develop an approach that considers a combination of

e-mail: voroninslava@gmail.com 
several parameters: color channels and the local and global nature of image processing.

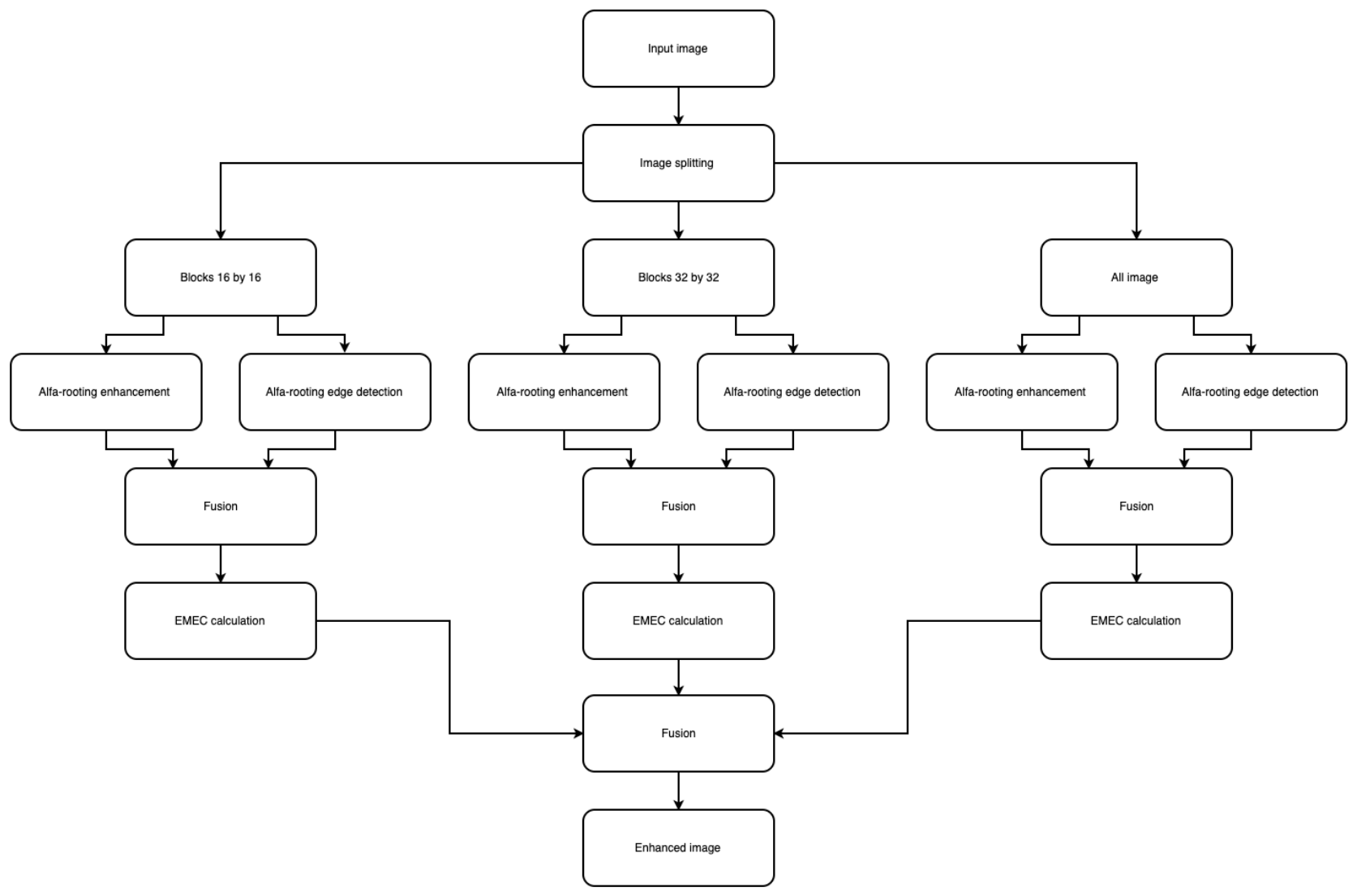

Fig. 1. Block diagrams of the proposed enhancement algorithm.

In this paper we proposed a novel enhancement technique using local and global processing and quaternion-based approach.

\section{Modified local and global contrast enhancement algorithm}

We consider a full model for representation and processing color images in the quaternion algebra. Color images are presented in the threefold complex plane where a complex image describes each color component. The color images of the RGB type store three colors in each pixel (red, green, and blue). For enhancement tasks, the image is usually converted to grayscale, thereby losing important information about color, saturation, and other important information associated color. Components of a color image can be represented as a quaternion $Q$. It is usually described using the form, where the basic algebraic form for a quaternion $q \in \mathbb{H}$ is $[13,14]$ :

$$
Q=q_{0}+q_{1} i+q_{2} j+q_{3} k,
$$

where $q_{0}, q_{1}, q_{2}, q_{3} \in \mathbb{R}$, the field of real numbers, and $i$, $j, k$ are three imaginary numbers. $\mathbb{H}$ can be regarded as a 4-dimensional vector space over $\mathbb{R}$ with the natural definition of addition and scalar multiplication. Each pixel for the color image can be regarded as a pure quaternion with zero real part [9].
The block diagram of the proposed enhancement algorithm for the color image is present in Fig. 1.

We split image in moving windows on disjoint blocks with different sizes (16 by 16, 32 by 32 and, i.e.) (Fig. 2) [15]. For every sub-image, we use the frequency domain enhancement method based on quaternion $\alpha$ rooting [13]. We perform image transformation, which needs to be enhanced, then manipulated the transform Discrete Fourier Transform (DFT) coefficients, and then perform the inverse orthogonal transform (Fig. 3).
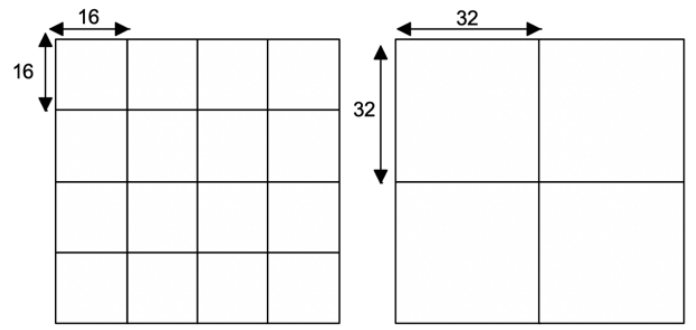

Fig. 2. Image splitting.

So, for every block, we use the transform-based enhancement algorithm base on the $\alpha$-rooting and magnitude reduction method [14]:

$$
\begin{gathered}
\left\{f_{n, m}\right\} \rightarrow\left\{F_{p, s}=\left|F_{p, s}\right| e^{\left.-j \theta_{p, s}\right\}} \rightarrow \mathrm{M} \circ F \rightarrow\left\{\hat{F}_{p, s}=M\left[\left|F_{p, s}\right|\right] e^{\left.-j \theta_{p, s}\right\}} \rightarrow\left\{\hat{f}_{n, m}\right\} .\right.\right. \\
\left|F_{p, s}\right| \rightarrow M\left[\left|F_{p, s}\right|\right]=\left|F_{p, s}\right|^{\alpha}, \quad \alpha \in(0,1)
\end{gathered}
$$


where $\alpha$ is a user defined operating parameter.

The $\alpha$-rooting transform depends on the parameter $\alpha$. We applied $\alpha$-rooting method two times. On the first step we are choosing the best (optimal) enhancement image through optimization of enhancement measure of the color image (EMEC) introduced by Agaian [13]:

$$
E M E C_{k_{1}, k_{2}}=\frac{1}{k_{1} \cdot k_{2}} \sum_{l=1}^{k_{1}} \sum_{k=1}^{k_{2}} 20 \cdot \log \frac{\max _{k, l}\left\{f_{R}, f_{G}, f_{B}\right\}}{\min _{k, l}\left\{f_{R}, f_{G}, f_{B}\right\}},
$$

where $\max _{k, l}$ and $\min _{k, l}$ respectively are the minimum and maximum of the image $f$ inside the block $\omega_{k, l}$.

On the second step, we are using the $\alpha$-rooting edge detection method. For this purpose, the parameter alfa set 0,2 .

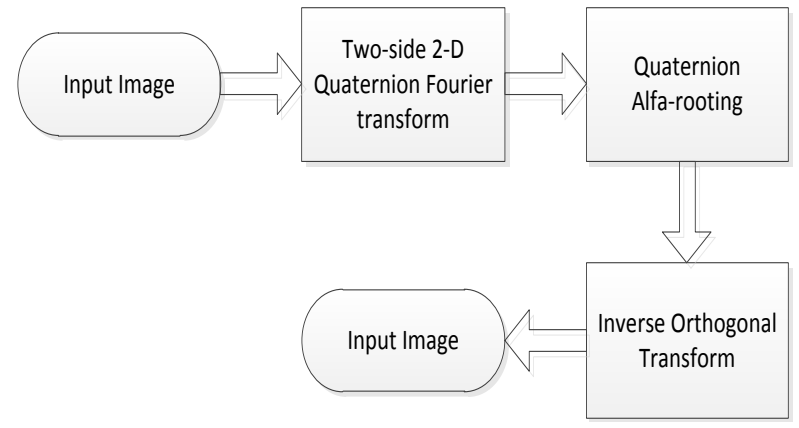

Fig. 3. Quaternion alfa-rooting image enhancement algorithm.

We calculate EMEC for every enhanced image and calculate weights, as follow:

$$
\begin{gathered}
W_{\tilde{f}_{1}}=\frac{E M E C_{\tilde{f}_{1}}}{E M E C_{\tilde{f}_{1}}+E M E C_{\tilde{f}_{2}}+E M E C_{\tilde{f}_{3}}+E M E C_{\tilde{f}_{4}}}, \\
W_{\tilde{f}_{2}}=\frac{E M E C_{\tilde{f}_{2}}}{E M E C_{\tilde{f}_{1}}+E M E C_{\tilde{f}_{2}}+E M E C_{\tilde{f}_{3}}+E M E C_{\tilde{f}_{4}}}, \\
W_{\tilde{f}_{3}}=\frac{E M E C_{\tilde{f}_{3}}}{E M E C_{\tilde{f}_{1}}+E M E C_{\tilde{f}_{2}}+E M E C_{\tilde{f}_{3}}+E M E C_{\tilde{f}_{4}}}, \\
W_{\tilde{f}_{4}}=\frac{E M E C_{\tilde{f}_{4}}}{E M E C_{\tilde{f}_{1}}+E M E C_{\tilde{f}_{2}}+E M E C_{\tilde{f}_{3}}+E M E C_{\tilde{f}_{4}}} .
\end{gathered}
$$

The resulted enhanced images define as:

$$
\tilde{f}=\tilde{f}_{1} \cdot W_{\tilde{f}_{1}}+\tilde{f}_{2} \cdot W_{\tilde{f}_{2}}+\tilde{f}_{3} \cdot W_{\tilde{f}_{3}}+\tilde{f}_{4} \cdot W_{\tilde{f}_{4}} \cdot
$$

\section{Experimental results}

In this section, we compare our results with well-known algorithms HE and CLAHE on the color satellite images from dataset AID. Figures 5-9 demonstrate the color image enhancement results obtained by the proposed algorithm ( $\mathrm{a}$ - original image; $\mathrm{b}$ - the enhanced image by the proposed method). The results achieved by the current proposed scheme have visually more contrast.

The proposed approach shows more details in the obtained enhanced color images. All details are either preserved or emphasized in the images. The color artifacts are more visible on the images processed by the approaching, which used secretly color channel processing. The experimental results (Table 1) show that the original images have the highest EMEC.

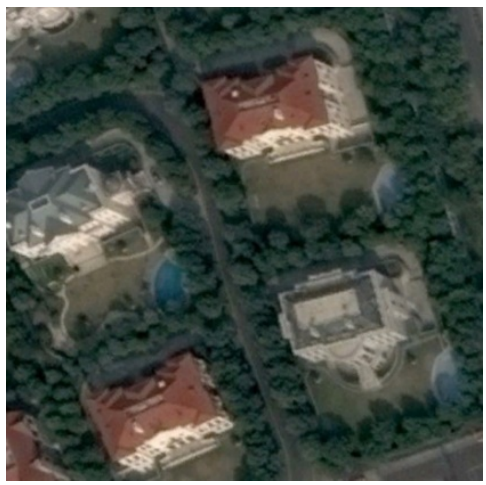

a)

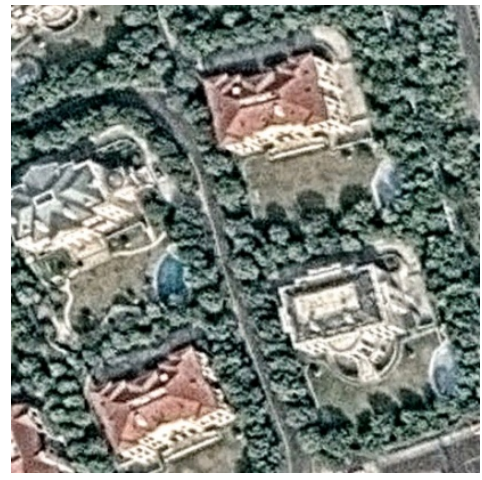

b)

Fig.4. Enhancement results for the Image \#1.
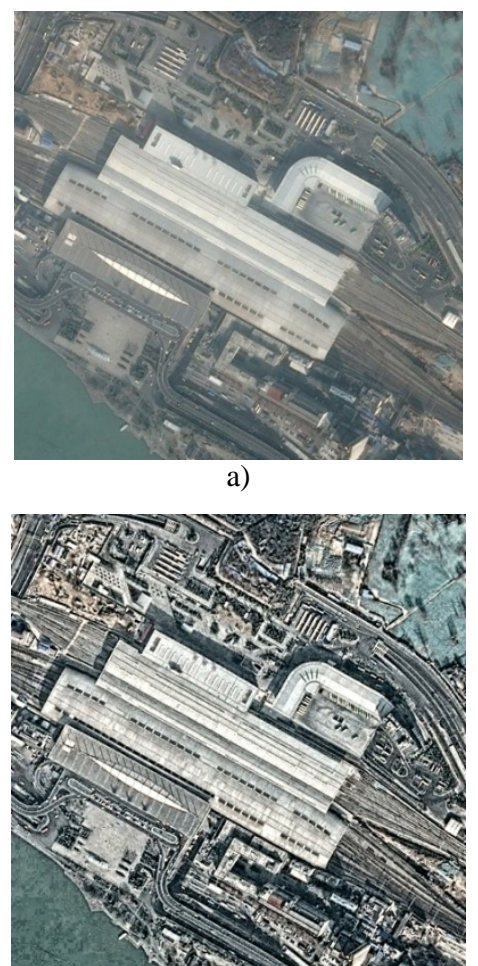

b)

Fig. 5. Enhancement results for the Image \#2. 


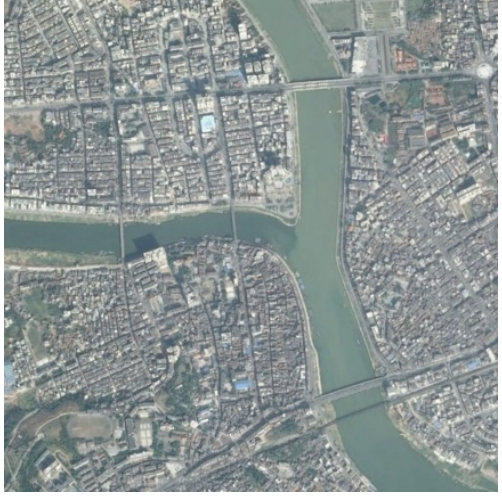

a)

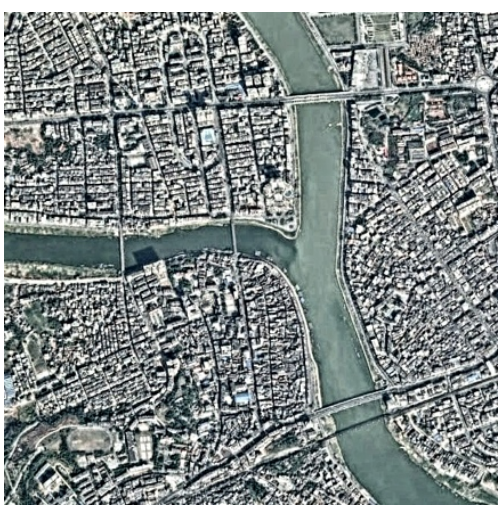

b)

Fig. 6. Enhancement results for the Image \#3.

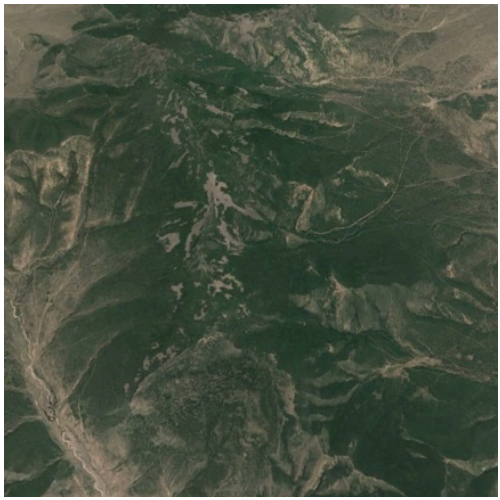

a)

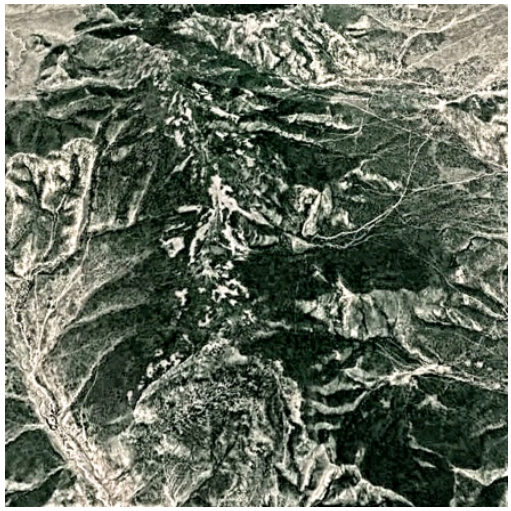

b)

Fig. 7. Enhancement results for the Image \#4.

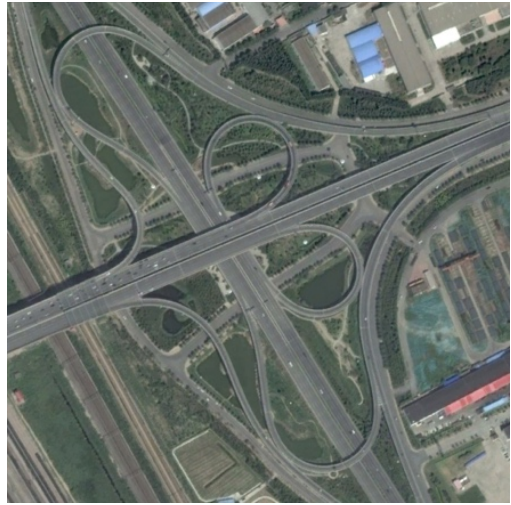

a)

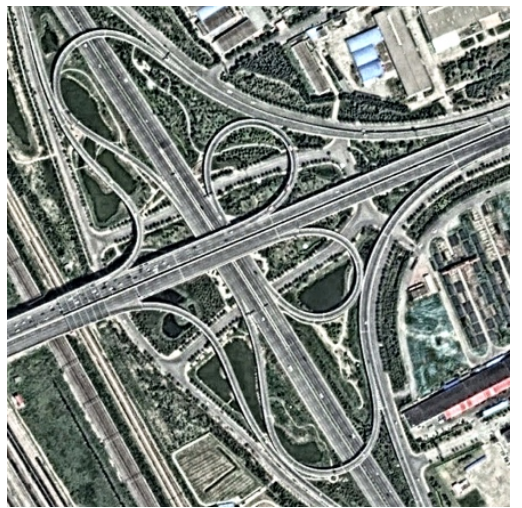

b)

Fig. 8. Enhancement results for the Image \#5.

Table 1. Comparison of resulting EMEC'S of different enhancement methods

\begin{tabular}{|l|c|c|c|c|}
\hline Images & Original & HE & CLAHE & $\begin{array}{c}\text { Proposed } \\
\text { method }\end{array}$ \\
\hline Image \#1 & 12,64 & 40,17 & 18,82 & $\underline{\mathbf{7 1 , 4 2}}$ \\
\hline Image \#2 & 13,09 & 56,38 & 26,52 & $\underline{\mathbf{1 1 9 , 0 2}}$ \\
\hline Image \#3 & 22,47 & 95,75 & 37,07 & $\underline{\mathbf{1 6 9 , 3 9}}$ \\
\hline Image \#4 & 14,25 & 59,46 & 22,95 & $\underline{\mathbf{1 3 0 , 2 9}}$ \\
\hline Image \#5 & 11,48 & 61,49 & 24,77 & $\underline{\mathbf{1 0 8 , 0 8}}$ \\
\hline
\end{tabular}

\section{Conclusion}

We introduce a novel quaternionic frequency enhancement algorithm which can combine the color channels and the local and global image processing. The basic idea is to apply $\alpha$-rooting image enhancement approach for blocks on the image with different size. The block size and alfa-rooting parameters driven through optimization using the Agaian's cost function - EMEC (color image enhancement non-reference quality measure). We use the a-rooting method of image enhancement in the subspace of quaternion numbers. The concept of the 2-D discrete quaternion Fourier transform (DQFT) is used to perform the a-rooting of color images 
(in RGB format). Some presented experimental results illustrate the performance of the proposed approach on color images in comparison with the state-of-the-art methods.

Acknowledgments. This work was supported by the Russian Science Foundation under grant №18-71-00137. We also thank the Center of Collective Use of MSUT "STANKIN" for providing resources.

\section{References}

1. H.J. Trussell, E. Saber, and M. Vrhel, IEEE Signal Processing Magazine, 22(1), 14-22 (2005).

2. K. Liang, Y. Ma, Y. Xie, B. Zhou and R. Wang, Inf. Physics. Tech, 55, 309-315 (2012).

3. T. Celik, IEEE Trans. Image Process, 23, 52985308 (2014).

4. M. Kanmani and V. Narsimhan, Multi To. Applications, 77, 23371-23387 (2018).

5. L. Zhang, L. Zhang, X. Mou and D. Zhang, IEEE Trans. Image Process, 20, 2378-2386 (2011).

6. S. Jenifer, S. Parasuraman and A. Kadirvelu, App Soft. Comp., 42, 167-177 (2016).

7. F. Hossain, M.R. Alsharif, International Conference on Convergence Information Technology, 1439-1444 (2007).

8. K. Zuiderveld, In: P. Heckbert: Graphics Gems IV, Academic Press, (1994).

9. J. Majumdar, S. Kumar, International Journal of Computer Engineering and Technology (IJCET), 11, 32-47 (2014)

10. X. Qiao, J. Bao, H. Zhang, L. Zeng, \& D. Li, Information processing in agriculture, 3, 206-213 (2017).

11. Z. Bichao, W. Yiquan, International Conference on Intelligent Human-Machine Systems and Cybernetics, 313-316 (2010).

12. S.S. Agaian, B. Silver, K.A. Panetta, IEEE transactions on image processing, 3, 741-758 (2007).

13. A.M. Grigoryan, J. Jenkinson, S.S. Agaian, Signal Processing, 109, 269-289 (2015).

14. A.M. Grigoryan, and S.S. Agaian, Applied Mathematics and Sciences: An International Journal (MathSJ), 1(3), (2014).

15. V. Voronin, E. Semenishchev, M. Ponomarenko, S. Agaian, Electronic Imaging, Image Processing: Algorithms and Systems XVI, 1-5 (2018). 